\title{
$K R A S$ gene mutation quantification in the resection or venous margins of pancreatic ductal adenocarcinoma is not predictive of disease recurrence
}

\section{Samuel Amintas}

Universite de Bordeaux College Sciences de la Sante

\section{Benjamin Fernandez}

CHU de Bordeaux: Centre Hospitalier Universitaire de Bordeaux

\section{Alexandre Chauvet}

CHU de Bordeaux: Centre Hospitalier Universitaire de Bordeaux

\section{Laurence Chiche}

Universite de Bordeaux College Sciences de la Sante

\section{Christophe Laurent}

Universite de Bordeaux College Sciences de la Sante

\section{Geneviève Belleannée}

CHU de Bordeaux: Centre Hospitalier Universitaire de Bordeaux

\section{Marion Marty}

CHU de Bordeaux: Centre Hospitalier Universitaire de Bordeaux

\section{Etienne Buscail}

CHU Toulouse: Centre Hospitalier Universitaire de Toulouse

\section{sandrine dabernat ( $\nabla$ sandrine.dabernat@u-bordeaux.fr)}

Universite de Bordeaux College Sciences de la Sante https://orcid.org/0000-0001-8372-9945

\section{Research Article}

Keywords: pancreatic ductal adenocarcinoma, molecular residual disease, KRAS, digital PCR

Posted Date: July 16th, 2021

DOI: https://doi.org/10.21203/rs.3.rs-674605/v3

License: (1) This work is licensed under a Creative Commons Attribution 4.0 International License. Read Full License 
Version of Record: A version of this preprint was published at Scientific Reports on February 22nd, 2022. See the published version at https://doi.org/10.1038/s41598-022-07004-x. 


\section{Abstract}

Pancreatic ductal adenocarcinoma (PDAC) patients eligible for curative surgery undergo unpredictable disease relapse. Even patients with good pathological response after neo-adjuvant treatment (NAT) remain susceptible to recurrent PDAC. Molecular analysis of R0 margins may identify patients with worse prognosis. Retrospective analysis of patients, either eligible for up-font surgery (UFS) or resected patients who underwent NAT with good pathological responses, collected survivals post-surgery and examined the molecular status of mutant KRAS by droplet digital PCR. Expectedly, KRAS mutant allele frequencies (MAF) were higher in UFS than in NAT tumor tissues or remnants, and in the ypT1 than the ypT0 tumors in the NAT group. Mutant KRAS positivity or MAFs in margins did not identify patients with detectable pathological disease in the NAT group, nor was it predictive of shorter recurrence-free or overall survival within or between groups. KRAS-double positivity in both venous and tumor resection margins did not identify patients with worse prognosis, regardless of the groups. The cohorts 'sizes were small due to limited numbers of patients meeting the inclusion criteria, but KRASpositivity or MAFs in resection and venous margins did not carry prognostic value. Comparison of margins from good versus bad responders receiving NAT may provide better clinical value.

\section{Introduction}

Pancreatic adenocarcinoma (PDAC) is a lethal cancer with limited efficacy of current treatments. This unfavorable situation is worsened by late diagnostic due to asymptomatic tumor development and unspecific symptoms. A vast majority of patients (85\%) suffer from locally advanced tumors and/or metastatic disease [1].

Surgery is the sole curative treatment but only $15 \%$ of patients present up-front resectable cancer. Moreover, even when resection is possible and complete, numerous patients relapse, without possible identification before surgery by predictive markers [2]. Two populations of patients can undergo surgical resection. First, patients eligible for up-front surgery carry tumors with no vascular contact. The second population is brought to surgery by neoadjuvant therapy (NAT) of borderline and locally advanced diseases, with better survival [3] compared to up-front resectable patients [4].

Current post-surgery tools are insufficient to both predict the course of the disease and estimate survival probability. For resected pancreatic cancers, tissue analysis carries prognostic value, in particular the histological subtype and the presence of vascular and/or perineural invasion [5], [6]. However, only $38 \%$ of T1/2NOR0 are disease-free 5 years after the diagnosis. In the same way, $28 \%$ of ypTONORO tumors relapse 2 years after surgery [7]. Carcinogenic antigen 19-9 (CA19-9) has post-surgery prognostic value [8]-[10]. However, its prognostic value was originally established on heterogeneous populations receiving gemcitabine monotherapy while the standard of care has now evolved to combined regimens (FOLFIRINOX or gemcitabine + nab-paclitaxel). It also suffers from a pretty high incidence of falsenegatives, since up to $10 \%$ of the general population does not express this marker. CA19-9 may be falsely elevated with biliary obstruction, biliary endoprotheses, and/or cirrhosis. Drops in CA19-9 levels with 
current chemotherapies were predictive of longer progression free survivals (PFS) underlining its utility as a follow-up marker more than a prognostic marker at the time of diagnosis [11].

Mutations of the KRAS gene are present in $90-95 \%$ of pancreatic adenocarcinomas. It is the most frequent mutated gene in PDAC [12]. Constitutive activation of KRAS occurs after point mutations preferentially in the exons 12 and 13 , which both represent $>98 \%$ of all activating mutations [13]. Furthermore, studies suggested that the molecular detection of KRAS mutations in surgery margins on histological samples could represent a sensitive way to identify residual disease [14]. This approach recently found a decreased recurrence free survival rate when molecular margins were KRAS mutationpositive [15], [16]. More precisely, Turrini and al. studied 22 patients with resectable PDAC naïve of neoadjuvant therapy. All patient had R0 margins and $56 \%$ positive venous margins were detected using a quantitative real time polymerase chain reactive (PCR) technique with a possible underestimation of positive margins. Kim et al. enrolled largest populations of PDAC with and without neo-adjuvant therapy (NAT) and found a relationship between recurrence free survival and positive margins, but their definition included detection of KRAS mutations and R1 surgical resection. This definition may increase the recurrence rate in the positive margin group.

The aim of this study was to investigate the status of KRAS mutation by ultra-sensitive droplet digital PCR in the resection and venous margins on histological tumor samples with R0 resection of head PDAC. We hypothesized that the proportion of KRAS mutation-positive $\mathrm{R} 0$ resection or venous margins should be lower to undetectable in samples of patients receiving neoadjuvant therapy with good response, i.e. displaying a yPTONORO pathological response, as compared to RO tumors and venous margins from patients who underwent up-front surgery. In the same way we also asked whether lower residual disease in resection or venous margins identified good responders to NAT with longer progression free or overall survival.

\section{Methods}

\section{Patients' inclusion}

\section{Patients with resectable PDAC}

Cohort description was previously published [17], [18]. Briefly we enrolled patients eligible for pancreatic surgery diagnosis with resectable pancreatic cancer (up-front surgery group, UFS, $n=22$ ). Diagnostic and staging status were performed by thoraco-abdominal CT-scan and liver MRI (magnetic resonance imaging). The patient inclusion/exclusion flowchart is provided in Figure 1A. Only the population of PDAC patients was studied. The study was approved by the French Ministry of Research under the number 2016-A00431-50. The registered clinical trial (https://clinicaltrials.gov/ct2/show/NCT03032913? term=panc-ctc\&draw=2\&rank=1) was first published 2017-01-26 under the number NCT03032913. All patients provided written consents.

\section{Good responders to neoadjuvant treatment}


The patients were selected retrospectively at the Hepatobiliary Department of the Bordeaux University Hospital database including all patients undergoing pancreaticoduodenectomy (PD) of pancreatic ductal adenocarcinomas. The database was started in 2009 and was prospectively incremented since. All patients provided written consents and the study was declared and approved by the Bordeaux University Hospital ethic comity. Inclusion criteria were as follows: Patients with locally advanced and/or borderline pancreatic head tumor lesions, who had PD with or without portal vein resection depending on the locoregional invasion (patients undergoing surgery after neo-adjuvant treatment, NAT) and a ypT0/1N0 pathological response on the resected tumor residue (good responders). Patients with endocrine tumors or carcinomas of the bile or duodenal and ampullary ducts, tumor stages greater than I, displaying distant metastases and for whom limits of microscopically resection were invaded (R1) after pathological analysis were excluded (Figure 1B). Each patient received neoadjuvant induction chemotherapy followed by radio chemotherapy.

\section{KRAS mutation detection}

Detection of mutant KRAS alleles was carried out on tumor lesions or tumor residues for the NAT responder cohort and the matched resection and venous margins. After histological selection of the 3 areas of interest by a trained pathologist, DNA of each area of interest was extracted from a $1 \mathrm{~mm}$ punch made in the paraffin block with the Maxwell RSC DNA FFPE Kit (Promega, Charbonnières-les-Bains, France). Extracted DNA was eluted in 70 microliters of "nuclease free" water and DNA concentration was determined by fluorimetry with DS11FX automated system (DeNovix). Digital droplet PCR was performed on each DNA sample using the ddPCR KRASScreening Multiplex Kit (Bio-Rad, ville, France). The probes cover the 7 most common mutations of KRAS codons 12 and 13 (G12S, G12R, G12C, G12D, G12A, G12V and G13D). Eight different probes are labeled either with a HEX fluorochrome (wild-type probe) or with a FAM fluorochrome (mutant probes). For each reaction, 50ng of DNA, 10 microliters of 2x ddPCR Supermix for Probes (non dUTP) and 1 microliter of 20X multiplex primers / probes (FAM + HEX) were used and mixed in a total volume of 20 microliters. Droplets were generated with droplet generation oil for probes (Bio-Rad) within the QX200 Droplet Generator (Bio-Rad). An incubation of the droplets at $95^{\circ} \mathrm{C}$ was performed for 10 minutes, followed by 40 cycles of PCR (denaturation at $94^{\circ} \mathrm{C}$ for 30 seconds / annealing and elongation at $55^{\circ} \mathrm{C}$ for 1 minute) on a C-1000 Touch Thermal Cycler (Bio-rad). During all of the PCR steps, the increment of the temperature ramp is $2.5^{\circ} \mathrm{C}$. The droplet fluorescence analysis was carried out using a QX200 droplet reader (Bio-Rad) and data analysis with the Quantasoft Analysis Pro software (Bio-rad).

\section{Follow-up}

After surgery, patients underwent the outpatient visit 1 month after the surgery, then every 3 months for the first 2 years, and every 6 months thereafter. Recurrence-free survival (RFS) was defined as time from resection of PDAC to the first radiologic recurrence or death due to PDAC. Overall survival (OS) was defined as time from the date of diagnosis until death due PDAC. 


\section{Statistical analysis}

Categorical variables were expressed as numbers and percentages. Continuous variables were expressed as means and standard deviations. A chi-squared test was used to compare categorical variables, and a non-parametric Mann-Whitney U test to compare continuous variables. Fisher's exact test was used to compare proportions of positive margins. Survival rates were calculated by the Kaplan-Meier method, and statistical significance was examined by the log-rank test and a Cox proportional hazards regression model. All statistical analyses were performed using GraphPad Prism Version 5.03 (GraphPad Software, Inc., La Jolla, California, USA). $p<0.05$ was considered statistically significant.

\section{Results}

\section{Patient's characteristics}

The baseline characteristics of the cohorts (Figure 1) and pathology data are shown in Table 1. Briefly, the patients' mean age was $66 \pm 8.2$ years in the NAT group and $62 \pm 9.1$ years in the UFS group. In the NAT group $33 \%$ of patients $(n=6)$ presented pathological complete response and $67 \%(n=12)$ were classified T1 or T2 according to the eighth edition of the American Joint Committee on Cancer TNM staging system [19]. In the UFS group most tumors were T3 or T4 ( $88 \%$ of cases, $n=15)$ and a minority were $\mathrm{T} 2$ $(n=2,12 \%)$. Three patients $(17 \%)$ received adjuvant chemotherapy in the NAT group versus 12 patients $(71 \%)$ in the UFS group.

\section{Residual molecular disease in tumor tissues}

We evaluated whether molecular quantification of mutant KRAS paralleled the pathological response in the NAT group or was predictive of worse prognosis in both groups.

First, we determined that KRAS mutant allele frequency (MAF) positivity threshold was $1.02 \%$, using non tumor DNA. An example of positive and negative KRAS mutation detection is presented in Figure 2A. Positivity threshold was calculated as the mean $+3 S D$ of MAFs from 5 healthy pancreatic and 2 healthy duodenum tissue samples (a total of 7 KRAS wild type DNA controls, Figure 2B).

Next, we quantified KRASMAFs in tumor tissues from patients who underwent UFS, and found an average value of $23 \pm 20.5 \%$ (Figure $3 A$ ). In resected tumor tissues from patients receiving NAT, MAFs were significantly higher in the ypT1 group than the ypT0 group ( $p=0.04$; Figure $3 B$ ), as expected. Noticeably, and confirming the validity of the $1.02 \%$ positivity threshold, we observed a mean MAF value of $1.1 \pm 0.2 \%$ in the ypT0 group, close to the threshold. By contrast the average values of the ypT1 group were 3-fold higher $(3.5 \pm 2.62 \%)$, showing concordance between the pathological response and the molecular residual disease.

Thus, molecular detection and quantification of mutant KRAS in tumor tissues is in agreement with expected results. 


\section{Tumor and venous margin KRASMAF assessment}

Within the course of patient follow-up after PD, pathological R0 tumor or venous margins do not carry reliable prognostic value since the majority of patients undergo disease relapse [20]. We hypothesized that considering the fibrotic nature of PDAC, the identification of residual tumor cells after NAT is very challenging for the pathologist. It is even more difficult after UFS since tumor cells might blend into the surrounding healthy tissue. The same difficulties might arise during venous margin pathological analysis. We tested the possibility to assess residual tumor cells in tumor and venous margins by the molecular presence of KRAS mutations in the resected margins.

The average MAFs in the resected margins of UFS samples was $1.0 \pm 0.99 \%$, in agreement with the molecular positivity threshold (Figure 3C). However, detailed analysis showed that only 2 out of 17 tumor margins $(12 \%)$ had MAFs $>1.02 \%$, suggesting that most of the resection margins were tumor cell free, in agreement with the R0 status. Venous margins tended to have higher MAFs although without statistical difference compared to resection margins (mean MAF 2.08 $\pm 3.9 \%, p=0.07$, Figure 3D). Again, although 7 out $17(41 \%)$ of the venous margins were positive, no statistical difference was reached compared to the positive tumor margins $(p=0.26)$.

In the NAT group, the average MAF in the resected margins was $1.0 \pm 0.25 \%$ and 9 out 18 samples (50\%) were above the positivity threshold (Figure 3E). KRAS positivity in venous margins was $1.01 \pm 0.38 \%$ ( $p>0.05$ as compared to resected margins), and 5 out $18(28 \%)$ were above the positivity threshold. The mean MAFs in resection or venous margins were not different in the UFS and NAT groups (Figure 3F).

\section{Survival analysis based on residual molecular disease}

Mean MAFs or proportions of KRASpositivity in margins were not different in the UFS and in the NAT groups. We evaluated whether they were associated with patients' survival. The mean follow-up period was $33 \pm 17$ months (median $=27$ months; range: 7-64 months). First, the NAT group showed longer recurrence free survival (RFS) and overall survival (OS) as compared to the UFS group (58.9 vs 13.9 months, $p<0.01$ and 64.4 vs 21.2 months, $p<0.01$, respectively, Supplementary Figure 1 ).

Expectedly, in the UFS group, high KRASMAFs in tumor tissues tended to be associated with shorter recurrence free survival (RFS, 6.7 months for MAFs $>23.3 \%$ versus 16.5 months for MAFs $<23.3, p=0.09$, Supplementary Figure 2A), even if the analysis did not reach significance probably because of the small size of the cohort. This trend was less marked for overall survival (OS, median survival of 21.2 months for MAFs $>23.3 \%$ versus 18.9 months for MAFs $<23.3 \%, p=0.08$, Supplementary Figure $2 B$ ).

Combined analysis of both groups showed no statistical difference in the UFS for positive versus negative tumor margins $(p=0.13$; Figure $4 A)$ or for positive versus negative venous margins $(p=0.28$; Figure 4B). In the same way, negative margins did not correlate with longer OS (Figure 4C and 4D, $p=0.52$ for resection margins and $\mathrm{p}=0.17$ for venous margins). 
Double negative margins (i.e. patients for whom both margins were negative) did not correlate with longer RFS (Figure $4 \mathrm{E}, \mathrm{p}=0.5$ ) or OS (Figure $4 \mathrm{~F}, \mathrm{p}=0.12$ ).

Finally, in the NAT group, double pathological (ypT1) and margin (either resection or venous) positivity did not identify patients with shorter RFS as compared to single positives (Figure $\mathbf{4 G}, n=9$ patient, $p=0.86$ ) or OS (Figure $4 \mathrm{H}, \mathrm{p}=0.81$ ).

Together, these results show that the detection of molecular residual disease in the resection or venous margins of UFS or NAT PDAC patients does not carry prognostic value.

In the same way, we double-positive margins (resection and venous) or double-negative margins did not correlate with local or distant recurrence.

\section{Discussion}

The present study tested the hypothesis that despite the R0 status of resected PDACs, detectable residual disease, predictive of disease relapse, might be evidenced in the resected tissues by molecular identification of mutant KRAS. We examined the mutant KRAS status in tissue samples of patients with the best prognosis, i.e. the margins of RO resected tissues of patients who underwent UFS and of good responder patients who had surgery after NAT.

Even if $K R A S$ is mutated in $>90 \%$ of PDACs, the use of $K R A S$ mutation status as a prognostic marker largely depends on the capacity of the technique to detect KRAS mutant variants. Indeed, they occur preferentially in the $\mathrm{G} 12$ position (98\%) while G13 and Q61 positions count for $1 \%$ each [13] of all KRAS mutations. The G12 aminoacid substitutions arise from 6 variants. In our study, 100\% of the DNAs from resected tissues in which tumor remnants were expected (i.e. tumors for the UFS group and tumors of the ypT1 NAT sub-group) showed detectable KRAS mutations. Of note, $50 \%(3 / 6)$ of the ypT0 NAT sub-group displayed KRAS mutations, despite the fact that the primary tumors were supposedly cleared. Overall, we did not detect any KRAS mutation in only 1 ypT0 patient (3\%, undetectable MAF in tumor tissue remnant and in margins). This observation is agreement with KRAS mutation frequency in PDAC (90-95\% according to the studies, reviewed in [21]) combined with the capacity of our technique to detect $98 \%$ of the mutations (all G12 and G13 codons substitutions). Altogether, these observations suggest that even if tumor clearance is pathologically confirmed, KRAS mutations persist despite the NAT and the surgery. Although poorly described, micrometastases are supposed to exist, as early as the onset of the disease. Indeed, PDAC can produce circulating tumor cells (CTCs) with metastatic potential during the formation of the primary tumor, before malignancy can be detected by histological methods [22].

Patients with KRAS alterations have decreased OS, and specifically, the KRASG12D mutation confers a worse prognosis in comparison with other KRAS alterations [21], [23]. It would be interesting to identify $K R A S$ mutations in our cohorts and evaluate the link with prognosis. 
The main limit of our study is the small size of the cohorts. However, they are representative of bigger published cohorts, in terms of RFS and OS. Indeed, in agreement with published resected cohorts comparable to our UFS group (for example [24], with an OS of 22.1 months and RFS 11.0 months versus 21.2 months and 13.9 months, our study). The NAT group selected good responders (complete pathological response, ypT0/1/N0). Of all the patients examined during the period, they represented $5.6 \%$, which is in the same ranged as previously observed [25]. NAT yielded good performance (8 out 18, i.e. $44 \%$ of relapses, and a RFS and OS of 58.9 and 64.4 months, respectively), as expected in published results [26]. In addition, our cohort yielded $17.6 \%$ of ypT0/1/N0 after chemoradiotherapy, which is in agreement with published data [26]. Finally, as expected, RFS and OS in the UFS group were lower than in the NAT group. However, the NAT group selected patients with good response (ypT0/1/N0) to treatment, which enhanced artificially the difference in the prognosis compared to the UFS group recruited prospectively. We also limited our analysis to the good responders of NAT, and it would be of interest to assess the margins of the ypT3/4/NO/N .

Residual disease evaluated by KRAS mutation positivity in margins was previously evaluated. In the venous margins of 22 UFS patients, Turrini et al. detected KRAS mutations in $55 \%$ of histologically negative venous margins [15], which is similar to our finding here of $41 \%$ (7 out of 17) and to Kim et al.'s [14]. However, by contrast to the cited studies, our survival analysis did not stratify patients according to their margin positivity. Adjuvant treatment cannot explain survival discrepancies. Interestingly, Kim's retrospective cohort counted only $11 \%$ of T3 tumors for $89 \%$ of $\mathrm{T} 1 / \mathrm{T} 2$ and $57 \%$ of $\mathrm{N}^{+}$. In the same way, Turrini's patients were mainly T1/T2 (72.8\%) and 59\% $\mathrm{N}^{+}$were recruited retrospectively. Our UFS prospective cohort included $88 \%$ of T3 tumors and only $12 \%$ of T1/T2 tumors, for $100 \% \mathrm{~N}^{+}$. Thus, it seems that Kim and Turrini's cohorts are not comparable to ours, at least in terms of tumor grade, which might explain the different prognosis value of KRAS mutation presence in margins, which may be of clinical value for lower grade PDACs. More recently, Kim et al. found that patients showing a KRAS MAFs $>4.19 \%$ had shorter DFS and OS in resection margins, regardless of the R status. In fact, they included R1 margins (43\%), and it is not clear whether the $4.19 \%$ threshold was distinct from the positivity threshold [16], since they did not detail this point. It is possible that this threshold identifies the R1 margins, hence its prognostic value. We rarely detected MAFs $>4.19 \%$ ( 2 out of 70 determinations), probably because we did not include R1 margins. Kim's patients had $73 \%$ of $\mathrm{T} 1 / \mathrm{T} 2$ tumors and $73 \%$ of $\mathrm{N}^{+}$. It would be interesting to analyze the $27 \%$ of patients with higher tumor grades.

\section{Conclusions}

This work clarifies the prognosis significance of KRAS mutation-positivity in margins. When margins are positive for $\mathrm{T} 1 / \mathrm{T} 2 / \mathrm{N} 0$ tumors, it may carry prognostic value. $\mathrm{T} 3 / \mathrm{N}^{+}$tumors may be already too invasive for KRAS-positivity to distinguish worse prognosis. Overall, very few studies evaluated KRAS mutant margin positivity and small cohorts were tested. More prospective analyses, including the future standard NAT need to be carried out. Finally, unlike hematological cancers, for which residual disease can be 
molecularly followed-up, solid tumors, in particular PDACs need the development of sensitive molecular tools and the identification of residual disease markers.

\section{Declarations}

Acknowledgements: We thank Pr François Moreau-Gaudry and Dr. Aurélie Bedel for their scientific input in the study design.

Author contributions: SA: project conception, data collection, results analysis, manuscript writing; BF: result analysis, figure building, manuscript writing; $A C$ : project conception, data collection, manuscript approval; LC: project conception, manuscript approval; CL: project conception, manuscript approval; EB: project conception, data collection, results analysis, manuscript approval, SD: project conception, results analysis, manuscript writing

Additional information: The authors have no competing interest to declare. This work was funded by the "Fonds Patrick Brou de Laurière" and the "Ligue Régionale contre le cancer".

\section{References}

[1] C. Gromisch, M. Qadan, M. Albuquerque Machado, K. Liu, Y. Colson, and M. W. Grinstaff, "Pancreatic Adenocarcinoma: Unconventional Approaches for an Unconventional Disease," Cancer Res., vol. 80, no. 16, pp. 3179-3192, Aug. 2020, doi: 10.1158/0008-5472.CAN-19-2731.

[2] B. Zhou et al., "Early detection of pancreatic cancer: Where are we now and where are we going?," Int. J. Cancer, vol. 141, no. 2, pp. 231-241, Jul. 2017, doi: 10.1002/ijc.30670.

[3] P. Rawla, T. Sunkara, and V. Gaduputi, "Epidemiology of Pancreatic Cancer: Global Trends, Etiology and Risk Factors," World J. Oncol., vol. 10, no. 1, pp. 10-27, Feb. 2019, doi: 10.14740/wjon1166.

[4] F. I. Macedo et al., "Survival Outcomes Associated With Clinical and Pathological Response Following Neoadjuvant FOLFIRINOX or Gemcitabine/Nab-Paclitaxel Chemotherapy in Resected Pancreatic Cancer," Ann. Surg., vol. 270, no. 3, pp. 400-413, Sep. 2019, doi: 10.1097/SLA.0000000000003468.

[5] S. van Roessel et al., "International Validation of the Eighth Edition of the American Joint Committee on Cancer (AJCC) TNM Staging System in Patients With Resected Pancreatic Cancer," JAMA Surg., vol. 153, no. 12, p. e183617, Dec. 2018, doi: 10.1001/jamasurg.2018.3617.

[6] L. Cong et al., "Tumor size classification of the 8 th edition of TNM staging system is superior to that of the 7 th edition in predicting the survival outcome of pancreatic cancer patients after radical resection and adjuvant chemotherapy," Sci. Rep., vol. 8, no. 1, Art. no. 1, Jul. 2018, doi: 10.1038/s41598018-28193-4. 
[7] H. Kourie et al., "Characteristic and outcomes of patients with pathologic complete response after preoperative treatment in borderline and locally advanced pancreatic adenocarcinoma: An AGEO multicentric retrospective cohort," Clin. Res. Hepatol. Gastroenterol., vol. 43, no. 6, pp. 663-668, Nov. 2019, doi: 10.1016/j.clinre.2019.03.007.

[8] Q. Dong et al., "Elevated serum CA19-9 level is a promising predictor for poor prognosis in patients with resectable pancreatic ductal adenocarcinoma: a pilot study," World J. Surg. Oncol., vol. 12, p. 171, Jun. 2014, doi: 10.1186/1477-7819-12-171.

[9] H. Imaoka et al., "Post-adjuvant chemotherapy CA19-9 levels predict prognosis in patients with pancreatic ductal adenocarcinoma: A retrospective cohort study," Pancreatol. Off. J. Int. Assoc. Pancreatol. IAP Al, vol. 16, no. 4, pp. 658-664, Aug. 2016, doi: 10.1016/j.pan.2016.04.007.

[10] A. Azizian et al., "CA19-9 for detecting recurrence of pancreatic cancer," Sci. Rep., vol. 10, no. 1, Art. no. 1, Jan. 2020, doi: 10.1038/s41598-020-57930-x.

[11] S. Awad, A. M. Alkashash, M. Amin, S. J. Baker, and J. B. Rose, "Biochemical Predictors of Response to Neoadjuvant Therapy in Pancreatic Ductal Adenocarcinoma," Front. Oncol., vol. 10, 2020, doi: $10.3389 /$ fonc. 2020.00620 .

[12] P. Bailey et al., "Genomic analyses identify molecular subtypes of pancreatic cancer," Nature, vol. 531, no. 7592, Art. no. 7592, Mar. 2016, doi: 10.1038/nature16965.

[13] K. L. Bryant, J. D. Mancias, A. C. Kimmelman, and C. J. Der, "KRAS: feeding pancreatic cancer proliferation," Trends Biochem. Sci., vol. 39, no. 2, pp. 91-100, Feb. 2014, doi: 10.1016/j.tibs.2013.12.004.

[14] J. Kim et al., "Unfavourable prognosis associated with K-ras gene mutation in pancreatic cancer surgical margins," Gut, vol. 55, no. 11, pp. 1598-1605, Nov. 2006, doi: 10.1136/gut.2005.083063.

[15] O. Turrini, M. Gilabert, J. Ewald, V. Moutardier, J.-R. Delpero, and J.-L. lovanna, “Resectable pancreatic head adenocarcinoma: Is R0 resection an illusion? Genetic evaluation of venous resection margin affirmed unrecognized disease," J. Visc. Surg., vol. 154, no. 5, pp. 329-333, Oct. 2017, doi: 10.1016/j.jviscsurg.2017.05.009.

[16] S. J. Kim et al., "Prediction of Recurrence With KRAS Mutational Burden Using Ultrasensitive Digital Polymerase Chain Reaction of Radial Resection Margin of Resected Pancreatic Ductal Adenocarcinoma," Pancreas, vol. 48, no. 3, pp. 400-411, Mar. 2019, doi:

10.1097/MPA.0000000000001255.

[17] E. Buscail et al., "CD63-GPC1-Positive Exosomes Coupled with CA19-9 Offer Good Diagnostic Potential for Resectable Pancreatic Ductal Adenocarcinoma," Transl. Oncol., vol. 12, no. 11, pp. 13951403, Aug. 2019, doi: 10.1016/j.tranon.2019.07.009. 
[18] E. Buscail et al., "High Clinical Value of Liquid Biopsy to Detect Circulating Tumor Cells and Tumor Exosomes in Pancreatic Ductal Adenocarcinoma Patients Eligible for Up-Front Surgery," Cancers, vol. 11, no. 11 , Oct. 2019 , doi: 10.3390/cancers 11111656 .

[19] M. B. Amin et al., "The Eighth Edition AJCC Cancer Staging Manual: Continuing to build a bridge from a population-based to a more 'personalized' approach to cancer staging," CA. Cancer J. Clin., vol. 67, no. 2, pp. 93-99, Mar. 2017, doi: 10.3322/caac. 21388.

[20] A. McGuigan, P. Kelly, R. C. Turkington, C. Jones, H. G. Coleman, and R. S. McCain, "Pancreatic cancer: A review of clinical diagnosis, epidemiology, treatment and outcomes," World J. Gastroenterol., vol. 24 , no. 43 , pp. $4846-4861$, Nov. 2018 , doi: 10.3748/wjg.v24.i43.4846.

[21] L. Buscail, B. Bournet, and P. Cordelier, "Role of oncogenic KRAS in the diagnosis, prognosis and treatment of pancreatic cancer," Nat. Rev. Gastroenterol. Hepatol., vol. 17, no. 3, pp. 153-168, Mar. 2020, doi: 10.1038/s41575-019-0245-4.

[22] A. D. Rhim et al., "EMT and dissemination precede pancreatic tumor formation," Cell, vol. 148, no. 1-2, pp. 349-361, Jan. 2012, doi: 10.1016/j.cell.2011.11.025.

[23] C. A. Mclntyre et al., "Alterations in driver genes are predictive of survival in patients with resected pancreatic ductal adenocarcinoma," Cancer, vol. 126, no. 17, pp. 3939-3949, Sep. 2020, doi: 10.1002/cncr.33038.

[24] B. lelpo et al., "A comparative study of neoadjuvant treatment with gemcitabine plus nab-paclitaxel versus surgery first for pancreatic adenocarcinoma," Surg. Oncol., vol. 26, no. 4, pp. 402-410, Dec. 2017, doi: 10.1016/j.suronc.2017.08.003.

[25] S. Gillen, T. Schuster, C. Meyer Zum Büschenfelde, H. Friess, and J. Kleeff, "Preoperative/neoadjuvant therapy in pancreatic cancer: a systematic review and meta-analysis of response and resection percentages," PLoS Med., vol. 7, no. 4, p. e1000267, Apr. 2010, doi: 10.1371/journal.pmed.1000267.

[26] J. He et al., "Is a Pathological Complete Response Following Neoadjuvant Chemoradiation Associated With Prolonged Survival in Patients With Pancreatic Cancer?," Ann. Surg., vol. 268, no. 1, pp. 1-8, Jul. 2018, doi: 10.1097/SLA.0000000000002672.

\section{Table}




\begin{tabular}{|c|c|c|}
\hline Characteristics & $\begin{array}{l}\text { Neo-Adjuvant treatment group } \\
(\mathrm{n}=18)\end{array}$ & Up-front surgery group $(n=17)$ \\
\hline & No of patients (\%) & No of patients (\%) \\
\hline \multicolumn{3}{|l|}{ Age, y } \\
\hline$\leq 60$ & $4(22)$ & $2(12)$ \\
\hline$>60$ & $14(88)$ & $15(88)$ \\
\hline \multicolumn{3}{|l|}{ Sex } \\
\hline Male & $12(67)$ & $10(59)$ \\
\hline Female & $6(33)$ & $7(41)$ \\
\hline \multicolumn{3}{|l|}{ Differentiation } \\
\hline Well & $8(44)$ & $5(29)$ \\
\hline Moderate & $7(39)$ & $7(42)$ \\
\hline Poor & $3(17)$ & $5(29)$ \\
\hline \multicolumn{3}{|c|}{ Lymph node invasion } \\
\hline Yes & $0(0)$ & $17(100)$ \\
\hline No & $18(100)$ & $0(0)$ \\
\hline \multicolumn{3}{|l|}{ T category } \\
\hline TO & $6(33)$ & 0 \\
\hline $\mathrm{T} 1-\mathrm{T} 2$ & $12(67)$ & $2(12)$ \\
\hline T3-4 & 0 & $15(88)$ \\
\hline \multicolumn{3}{|c|}{ Adjuvant treatment } \\
\hline Yes & $3(17)$ & $12(71)$ \\
\hline No & $15(83)$ & $5(29)$ \\
\hline GEMCITABINE & $2(11)$ & $4(24)$ \\
\hline mFOLFIRINOX* & $1(6)$ & $13(76)$ \\
\hline
\end{tabular}

\section{Figures}


A

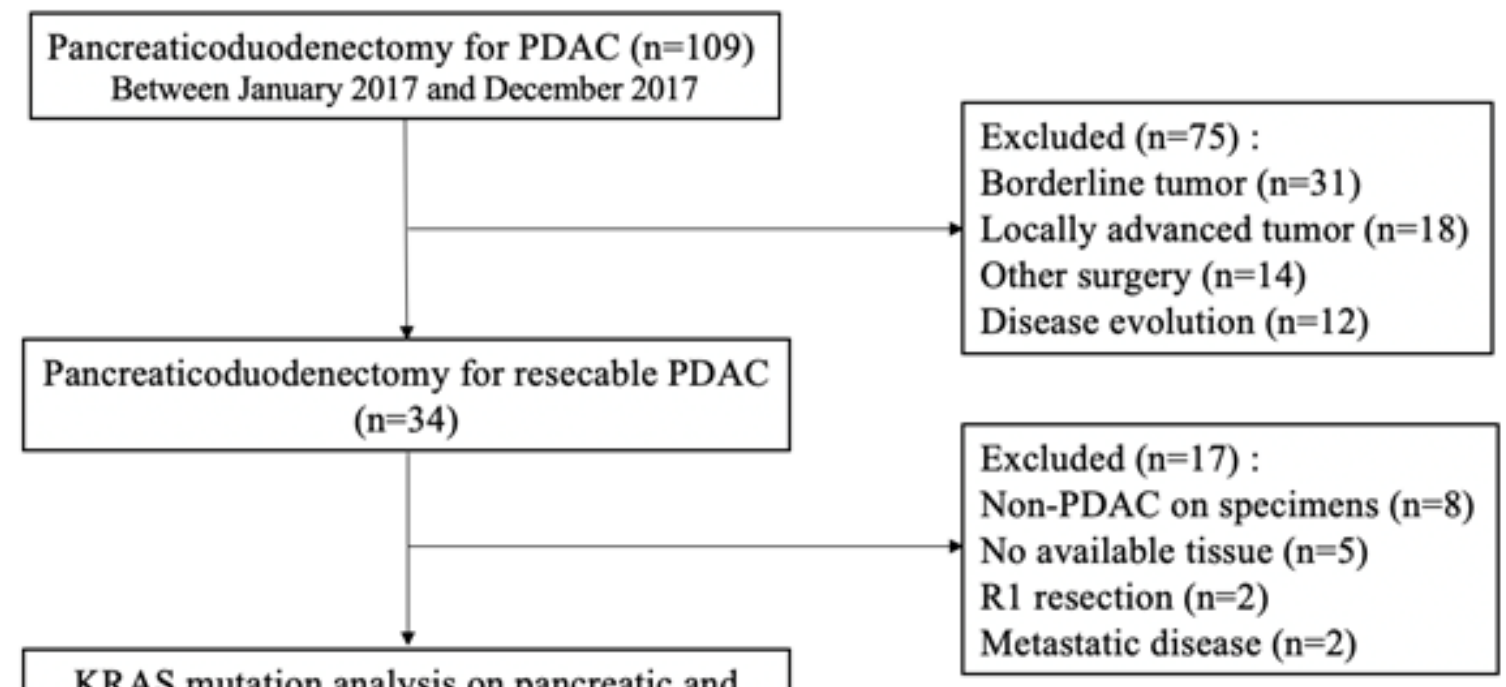

$\mathrm{B}$

Pancreaticoduodenectomy for PDAC $(\mathrm{n}=320)$

Between December 2009 and December 2017

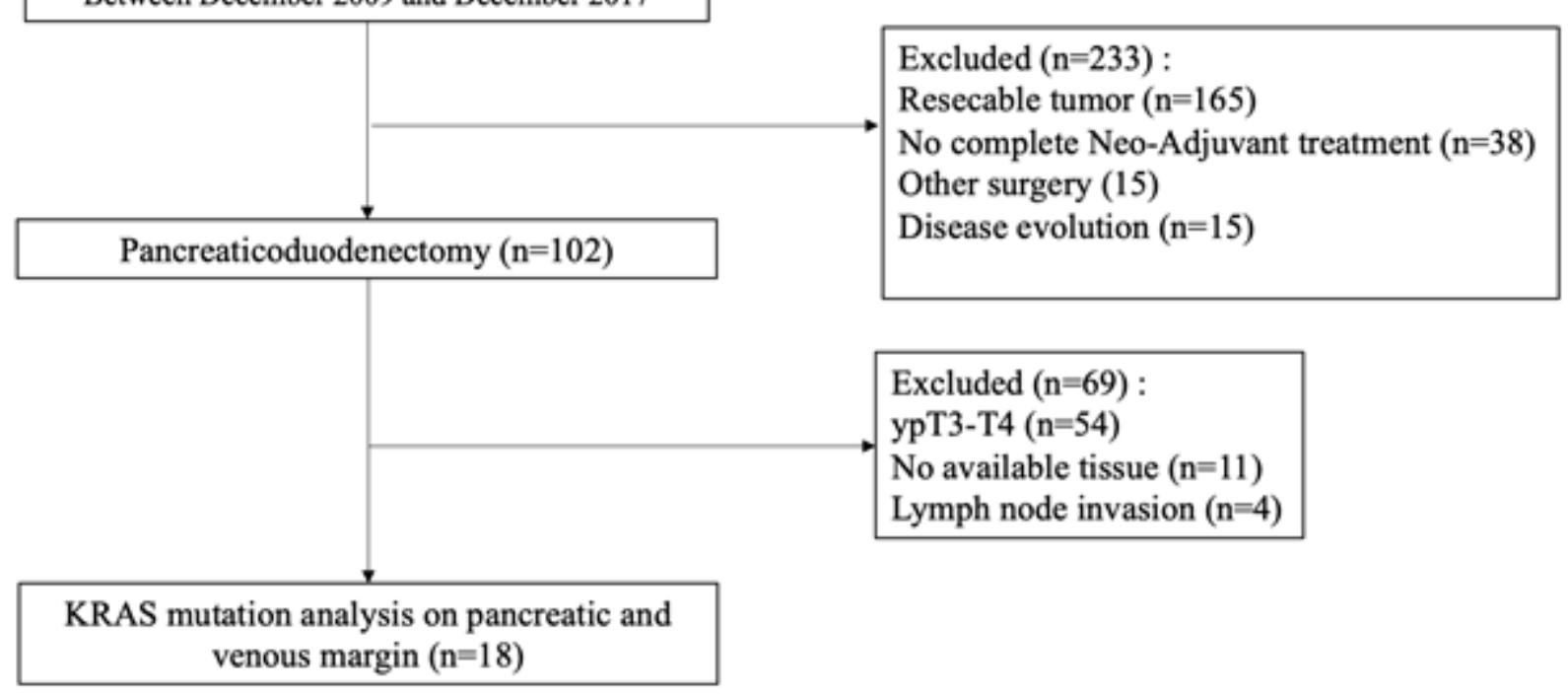

Figure 1

Flow chart of the cohorts. (A) Up-font surgery cohort (UFS, PANC-CTC \#NCT03032913), (B) Neo-Adjuvant treatment (NAT) good responders 
A

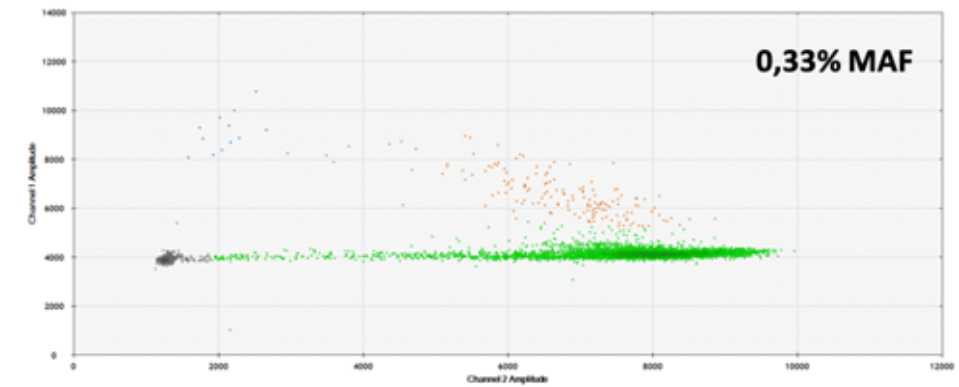

B

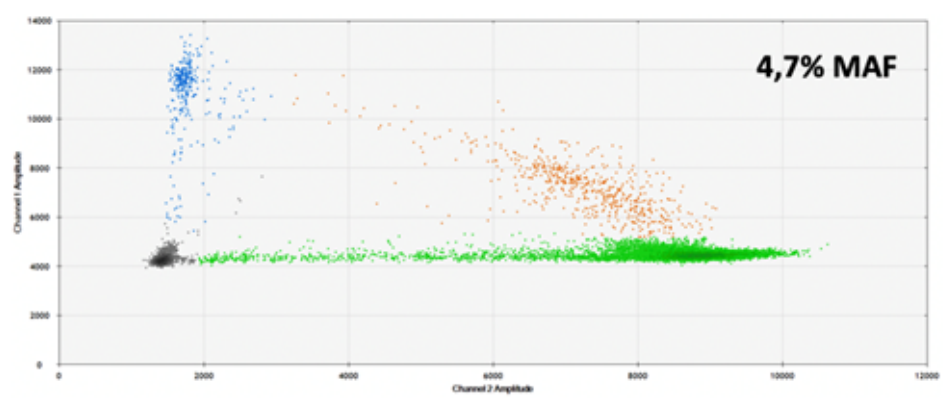

C

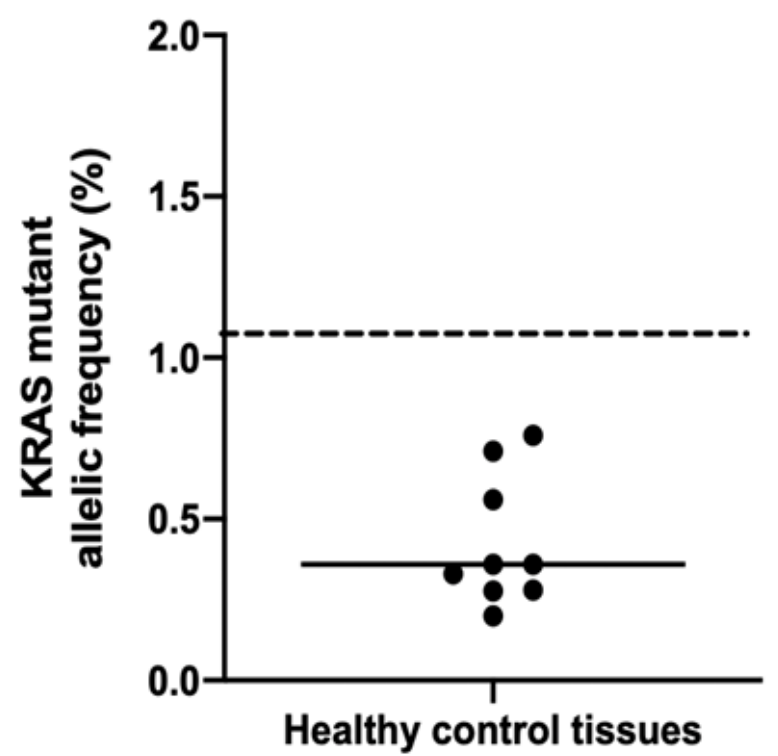

Figure 2

Assessment of molecular margin KRAS positivity threshold. (A) Droplet digital PCR Two-dimensional (2D) plot of a negative margin for KRAS G12/13 mutation (FA = 0,33\%). (B) Droplet digital PCR 2D plot of a positive margin for KRAS G12/13 mutation ( $F A=4,7 \%$ ). (C) KRAS mutant allele frequency of the 7 KRAS wild type DNA controls. Positivity threshold was calculated as the mean +3 SD of MAFs of the 7 samples $(1,02 \%)$. 
A

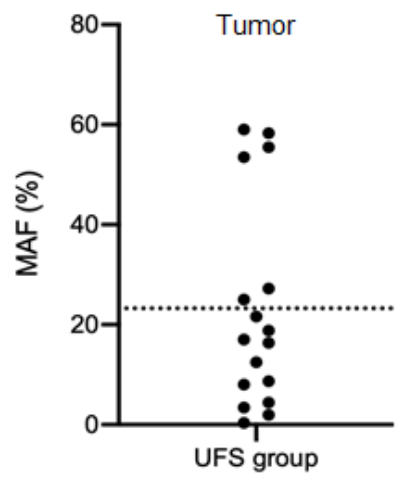

B

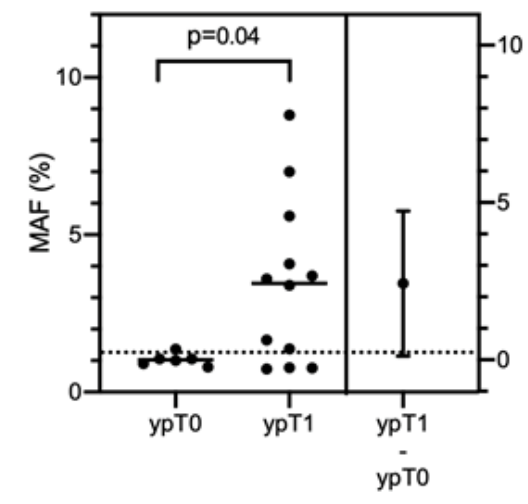

C

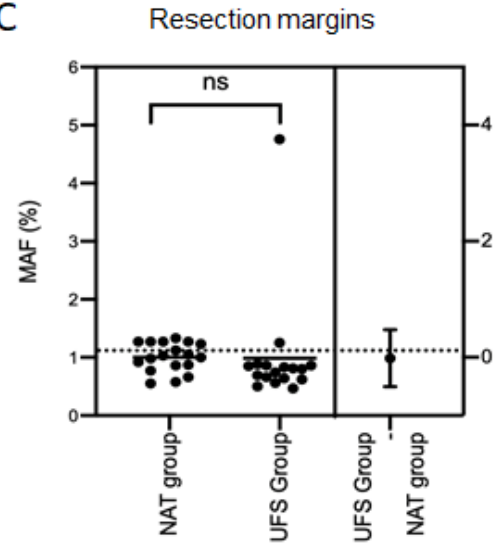

D Venous margins

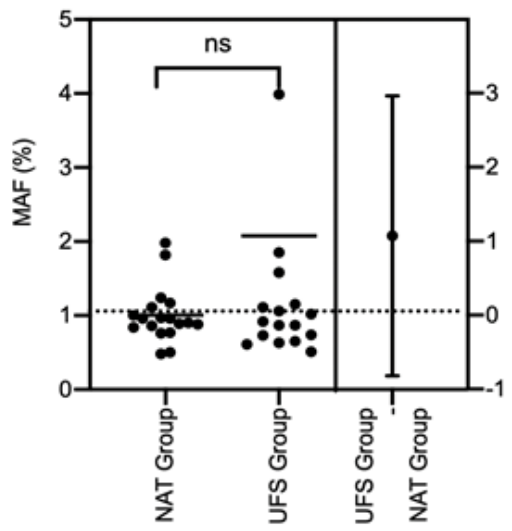

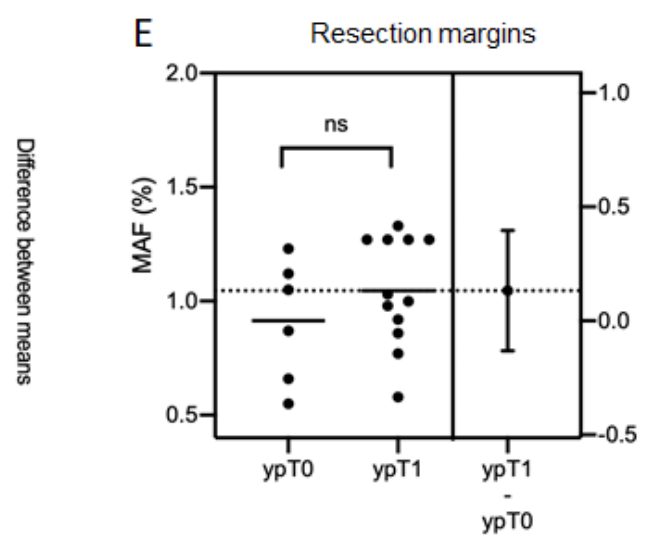

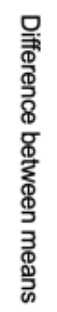

$\mathrm{F}$

F Venous margins

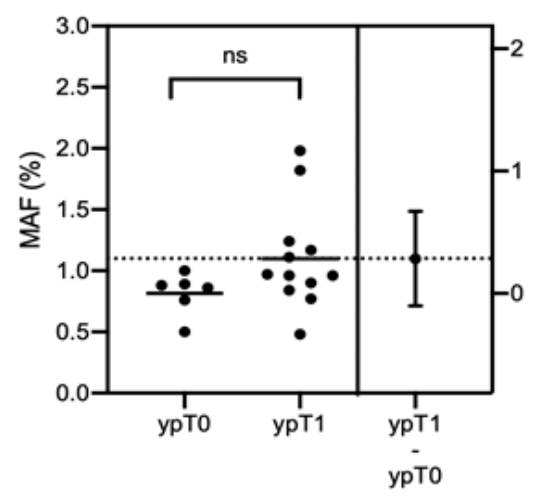

\section{Figure 3}

Molecular analysis of tumor tissues and margins. (A) KRAS MAFs in tumor tissues of UFS group (B) KRAS MAFs in tumor remnants of the NAT patients, showing significant difference in the tumor tissues between ypT0 and ypT1 tumors in the NAT Group ( $\mathrm{p}=0.04)$. KRAS MAFs in resection margins (C) and in venous margins (D) of NAT and UFS groups. KRAS MAFs in resection margins of the ypT0 and ypT1(E) and venous margins (F) from NAT group. 
A

Resection margins

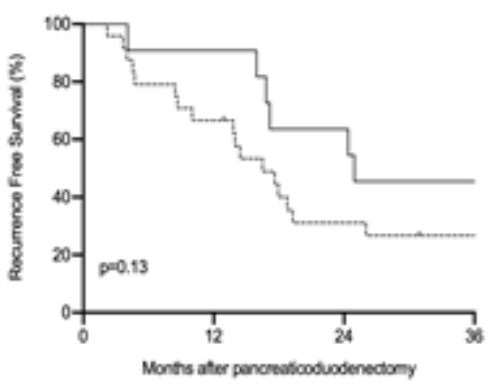

C

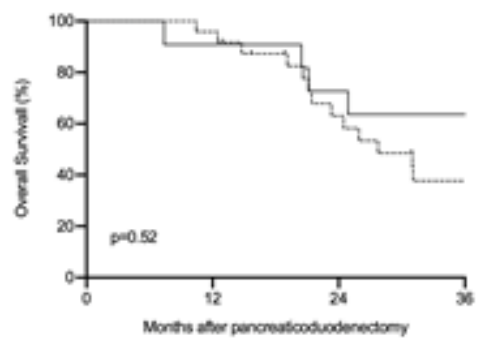

E

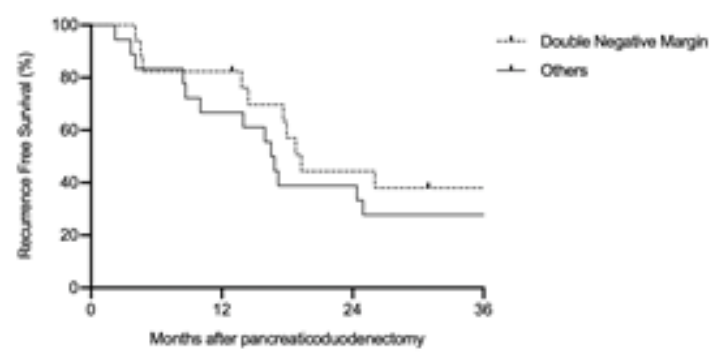

G

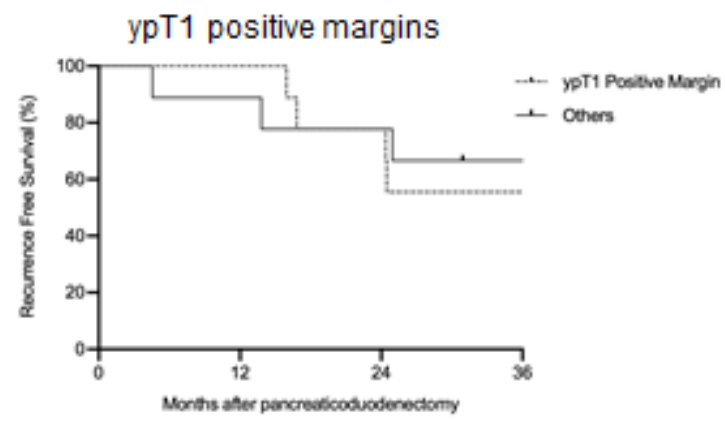

- Postove Margin

-.t. Negrtwo Margin

+ Poseve Marin

Months ator pencreutooduodenedomy
B Venous margins

-2.- Negaswe Margin

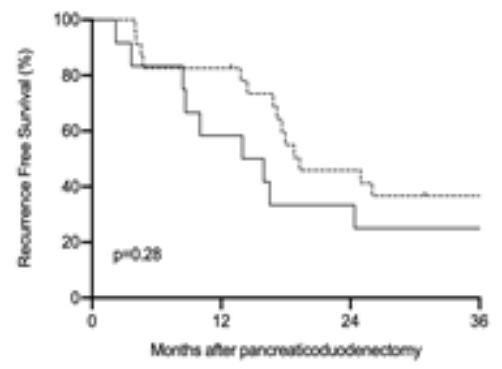

D

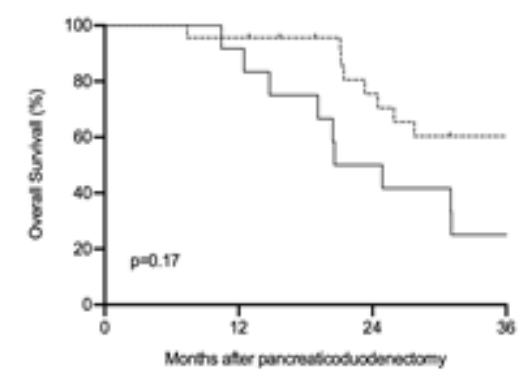

F

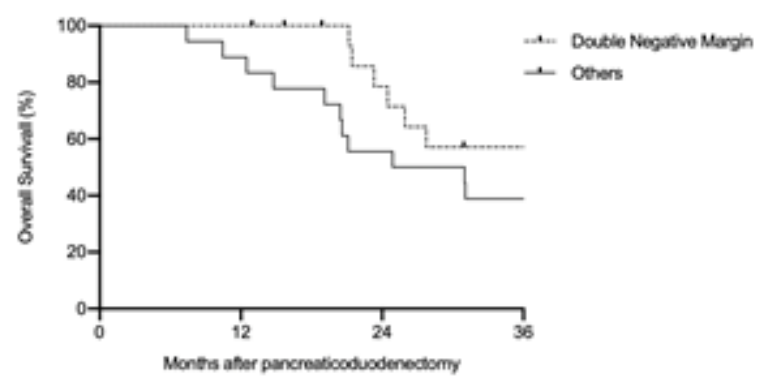

$\mathrm{H} \quad \mathrm{ypT1}$ positive margins

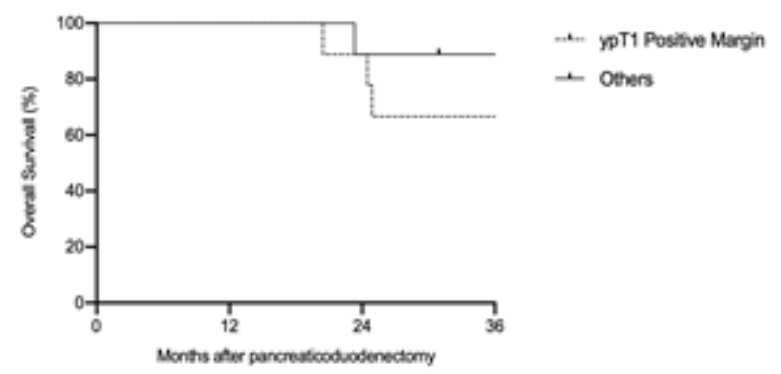

\section{Figure 4}

Survival analyses according to KRAS molecular status. (A) Recurrence free survival for resection marginpositive or -negative for KRAS mutations. (B) Recurrence free survival for venous margin-positive or negative for KRAS mutations. (C) Overall survival for resection margin-positive or -negative for KRAS mutations. (D) Overall survival for venous margin-positive or -negative for KRAS mutations. (E) Recurrence free survival for double negative margins as compared to single or double positives. (F) Overall survival for double negative margins as compared to single or double positives. (G) Recurrence 
free survival for ypT1venous and/or resection-positive margins. (H) Overall survival for ypT1venous and/or resection-positive margins.

\section{Supplementary Files}

This is a list of supplementary files associated with this preprint. Click to download.

- SupplementalFigure1 krasmarges.pptx

- SupplementalFigure2kras.pptx 